\title{
Sugars, fruits and fructose drinks
}

(Index words: Refined sugars, obesity, hypertriglyceridaemia, poor glycaemic control)

\section{Sugars, fruits and fructose drinks}

Fructose drinks are being promoted among diabetics. We write this piece to clarify certain nutritional issues about these drinks. With regard to human nutrition, sugars in foods can be divided into two groups: sugars in intact fruits and vegetables (intrinsic sugars) and sugars that are added to food and drinks by manufacturers, cooks or consumers (added sugars), e.g. sucrose, fructose. Sucrose (extracted from sugar cane) is a disaccharide which is converted into glucose and fructose in the intestines. Added sugars, honey (rich in fructose), syrups and fruit juices are collectively described as "free sugars". Several countries have developed guidelines to restrict intake of added sugar because of its health hazards. Dietary guidelines do not recommend the restriction of intrinsic sugars as they do not generally have adverse health outcomes.

Although there is no direct causal association between the consumption of free sugars and coronary heart disease, diabetes and other chronic diseases, they contribute substantially to the global epidemic of obesity and its consequences [1]. Sugars promote a positive energy balance. Total energy intake increases when energy density of the diet increases, whether by free sugars or fat. Drinks rich in free sugars increase the overall energy intake.

Fruits are a good source of vitamin C, pectin (a soluble fibre), carotene and potassium. In addition fruits provide fructose (a monosaccharide) as the main sugar and small amounts of sucrose and glucose [2]. Ripe fruits contain a greater proportion of sucrose and glucose compared to fruits that are not ripe. Fruits add variety to diet with many different natural tastes and flavours. Two or three daily servings of fruits are recommended in the diet. The soluble fibre in fruits helps to reduce serum cholesterol by reducing enterohepatic circulation of bile salts.

Diets rich in sucrose, and high fructose syrups used in the manufacture of food and beverages, lead to large amounts of fructose ( and glucose) entering the portal vein. Excess fructose accumulates in the liver and leads to problems such as fatty liver and hyperuricaemia. Fructose undergoes more rapid metabolism (glycolysis) in the liver than glucose, because it bypasses the key regulatory step in glycolysis [3]. This results in fructose flooding pathways in the liver, leading to increased triglyceride synthesis, very low density lipoprotein (VLDL) secretion and hypertriglyceridaemia. In addition, acute loading of the liver with fructose due to high intakes causes sequestration of phosphate during fructose -1phosphate formation. This leads to decreased levels of ATP, the energy currency of the cell. The accumulated ADP is catabolised to uric acid leading to hyperuricaemia. Decreased ATP synthesis can lead to impairment of hepatic function [3].

Fructose is not essential to the body, hence the question of a daily requirement of fructose in the diet does not arise. Fructose is the component in a fruit which gives calories that may lead to obesity. This is the reason why consumption of fruits should be limited to two or four servings a day. This does not mean that fruits should be avoided, but excess consumption should be curtailed.

We have observed that sachets of fructose are being promoted as a recommended drink for people with diabetes. In a fructose drink, the most beneficial components of fruit such as soluble fibre, vitamin $\mathrm{C}$ and natural taste are absent. Consumption of fructose drinks by people with diabetes could cause obesity, poor blood glucose control and hypertriglyceridaemia. Diabetics should avoid all refined sugars including sucrose and fructose. Even during convalescence, natural fruit juice provides the taste and colour without any additives, and it is easily digestible, e.g. orange juice. Rice conjee or vegetable soup with rice and dhal added are nutritious, easily absorbable liquids that provide calories with natural proteins and carbohydrates.

Fructose drinks are not recommended for people with diabetes and should not be a part of their diet.

\section{References}

1. Mann J. Sugar revisited-again. Bulletin of the World Health Organisation 2003; 81: 552.

2. Wikramanayake TW, ed. Food and Nutrition. 3rd edn. Colombo 7: Hector Kobbekaduwa Agrarian Research and Training Institute, 1996.

3. Champe PC, Harvey RA, eds. Lippincott's Illustrated Reviews: Biochemistry. 2nd edn. Philadelphia: JB Lippincott Company, 1994.

K de Abrew ${ }^{1}$, S Atukorale ${ }^{3}$, RL Jayakody ${ }^{2}$, P Galappaththy ${ }^{1}$, MSG de Silva ${ }^{4}$ and Colvin Goonaratna ${ }^{5},{ }^{1}$ Senior lecturer, ${ }^{2}$ Professor and Head, Department of Pharmacology, ${ }^{3}$ Professor and Head, Department of Biochemistry and Molecular Biology, Faculty of Medicine, Colombo; ${ }^{4}$ Physician, National Hospital of Sri Lanka, Colombo; and Editor, CMJ. Correspondence: K de A., e-mail: 271002@celltelnet.lk (Competing interests: All of us advise diabetes patients under our care to eschew commercial fructose drinks and other refined sugars). 